\title{
Proceedings
}

\section{25th Euromicro International Conference on Parallel, Distributed and Network-Based Processing PDP 2017}

St. Petersburg, Russia 6-8 March 2017 



\title{
Proceedings
}

\section{25th Euromicro International Conference on Parallel, Distributed and Network-Based Processing PDP 2017}

St. Petersburg, Russia 6-8 March 2017

\author{
Edited by
}

Igor Kotenko, Yiannis Cotronis and Masoud Daneshtalab 
Copyright (C) 2017 by The Institute of Electrical and Electronics Engineers, Inc.

All rights reserved.

Copyright and Reprint Permissions: Abstracting is permitted with credit to the source. Libraries may photocopy beyond the limits of US copyright law, for private use of patrons, those articles in this volume that carry a code at the bottom of the first page, provided that the per-copy fee indicated in the code is paid through the Copyright Clearance Center, 222 Rosewood Drive, Danvers, MA 01923.

Other copying, reprint, or republication requests should be addressed to: IEEE Copyrights Manager, IEEE Service Center, 445 Hoes Lane, P.O. Box 133, Piscataway, NJ 08855-1331.

The papers in this book comprise the proceedings of the meeting mentioned on the cover and title page. They reflect the authors' opinions and, in the interests of timely dissemination, are published as presented and without change. Their inclusion in this publication does not necessarily constitute endorsement by the editors, the IEEE Computer Society, or the Institute of Electrical and Electronics Engineers, Inc.

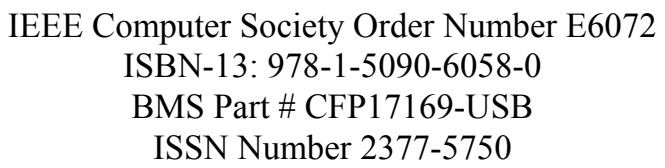

Additional copies may be ordered from:

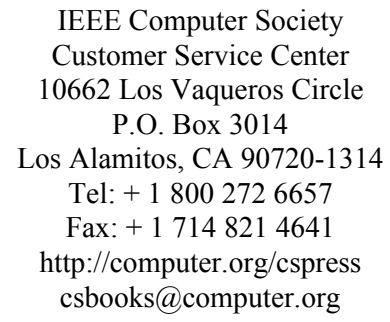

IEEE Computer Society Customer Service Center 10662 Los Vaqueros Circle P.O. Box 3014

Los Alamitos, CA 90720-1314

Tel: + 18002726657

Fax: + 17148214641

http://computer.org/cspress csbooks@computer.org

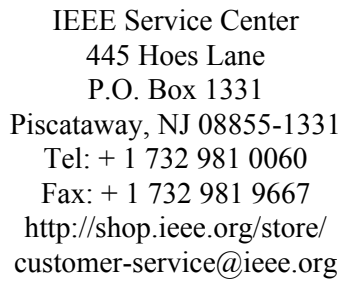

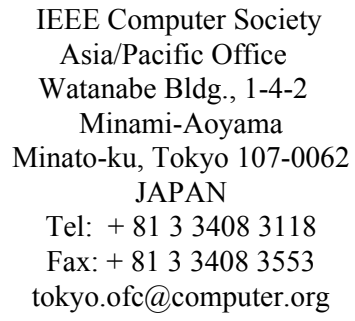

Individual paper REPRINTS may be ordered at: <reprints@computer.org>

Editorial production by Juan E. Guerrero

Cover art production by Mark Bartosik

Printed in the United States of America by Applied Digital Imaging

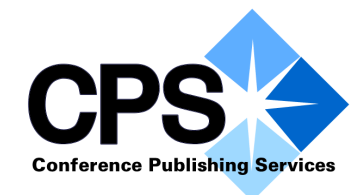

\section{IEEE Computer Society Conference Publishing Services (CPS)}

http://www.computer.org/cps 


\section{Message from the General Chairs PDP 2017}

Welcome to the 25th Euromicro International Conference on Parallel, Distributed, and Network-Based Processing (PDP 2017).

The topics of the Conference, Parallel, Distributed, and Network-Based Processing have undergone impressive changes over recent years. New architectures and applications have become the central focus of the discipline. These changes are often a result of mutual enrichment of parallel and distributed technologies with other rapidly evolving technologies. The PDP conference continues to have a distinctive composition: a main track invites papers over a broad range of topics, and Special Sessions focus on a particular sub-domain of research fields. Each Special Session has its own Chair(s) and Program Committee and invites and selects its own papers, all under the umbrella of the overall conference structure.

The high number of interesting and significant research papers submitted to PDP demonstrates that the conference is becoming an ever more important international event in the field of parallel and distributed computing research. This year PDP 2017, main track and special sessions, have received altogether 162 submissions of 459 authors from 40 countries. On average each paper received 3.1 reviews, with no paper receiving fewer than two reviews. The result was the selection of 52 regular and 41 short papers for publication in these proceedings. The acceptance rate of regular papers in the PDP 2017 including special sessions is $32 \%$ and $57 \%$ including short. In the main track we received 73 papers and accepted 22 as regular and 17 as short papers. The acceptance rate of regular papers in main track is $30 \%$ and including short papers $53 \%$.

We wish to thank all who contributed to the success of the event: the 459 authors, the 227 members of Programme Committees, the 59 sub reviewers, the Keynote speakers, Didier Elbaz, Vladimir Zaborovsky, Victor Toporkov and Vladimir Gorodetsky, and the 24 Special Session Chairs:

- Didier El Baz (GPU Computing and Many Integrated Core Computing);

- Simon Bliudze and Borzoo Bonakdarpour (Formal Approaches to Parallel and Distributed Systems);

- Daniele D'Agostino and Ivan Merelli (Advances in High-Performance Bioinformatics, Systemsand Synthetic Biology);

- Igor Kotenko (Security in Parallel, Networked and Distributed Systems);

- Sébastien Lafond, Laurent Lefèvre, Jean-Marc Pierson, Tim Süß and Tuan Trinh (Energy Efficient Management of Parallel Systems, Platforms, and Computations);

- Thierry Delaitre, Gabor Kecskemeti and Attila Kertesz (Cloud Computing on Infrastructure as a Service and its Applications);

- Donato D'Ambrosio, Georgios Sirakoulis, William Spataro, Giuseppe Trunfio (High Performance Computing in Modelling and Simulation);

- Nader Bagherzadeh, Masoud Daneshtalab, Masoumeh Ebrahimi and Hamid Sarbazi-Azad (OnChip Parallel and Network-based Systems);

- Karl-Erwin Grosspietsch and Konrad Klöckner for organizing the Work-in-Progress session.

We also thank Andrey Chechulin and Maxim Kolomeec for development and maintaining PDP 2017 web site; Euromicro Office staff for their efficient administration; Juan Guerrero and the team at Conference Publishing Services for producing these proceedings. Finally, we thank Marco Danelutto, 
Chairman of the Parallel and Distributed Processing Euromicro Committee and Karl-Erwin Grosspietsch, Chairman of Euromicro, for their support.

We welcome old and new friends and colleagues. We hope you all enjoy the scientific as well the social program in the tradition of the cordial spirit of PDPs.

Igor Kotenko, St. Petersburg Institute for Informatics and Automation of the Russian Academy of Sciences Yiannis Cotronis, National and Kapodistrian University of Athens

Masoud Daneshtalab, KTH Royal Institute of Technology

PDP 2017 Conference General Chairs 


\section{Message from the Organizing Committee Chairs PDP 2017}

On behalf of the Organizing Committee we are pleased to welcome you to the 25nd Euromicro International Conference on Parallel, Distributed, and Network-Based Processing (PDP 2017), organized by the Computer Security Problems Laboratory of the St. Petersburg Institute for Informatics and Automation of the Russian Academy of Sciences (SPIIRAS).

SPIIRAS was founded in 1978 on the basis of the Computer Science Department of Ioffe Physical and Technical Institute of the USSR Academy of Sciences. Currently the Institute is the only one scientific institution in the North-West region of Russia that does basic research in information technologies and automation. Since February, 1991 and at present Professor Rafael M. Yusupov, Corresponding Member of the Russian Academy of Sciences (RAS) is the head of the Institute. One of the first global computer and information networks in the country the Academic Network "North-West" was created. According to the Decree of the Russian Federation Government dated 12/30/ 2013 the Institute was turned over to the authority of the RF Federal Agency for Scientific Organisations (FASO). The Russian Academy of Sciences provides for the scientific and methodological guidance in regard to the Institute research activities. The Institute purpose and object of activities are to do basic, prediscovery and applied scientific research aimed at the acquisition of new knowledge in the area of informatics and automation; the development of control methods, and information and telecommunication technologies applicable to solving actual scientific and engineering as well as social and economic problems, including those of interdisciplinary nature.

St. Petersburg is Russia's second-largest city after Moscow and situated on the Neva River, at the head of the Gulf of Finland on the Baltic Sea. It was the imperial capital for two centuries; it was founded by tsar Peter the Great in 1703. It remains Russia's cultural center, with venues like the Hermitage, one of the largest art museums in the world, the State Russian Museum showcasing Russian art, from Orthodox icon paintings to Kandinsky works, and the Mariinsky Theatre hosting opera and ballet. The historic centre of St. Petersburg and related groups of monuments constitute a UNESCO World Heritage Site. St. Petersburg is a major trade gateway, financial and industrial centre of Russia specializing in oil and gas trade, shipbuilding yards, aerospace industry, radio and electronics, software and computers; machine building, heavy machinery and transport, mining, instrument manufacture, metallurgy, chemicals, pharmaceuticals, medical equipment, publishing and printing, food and catering, wholesale and retail, textile and apparel industries, and many other businesses.

Organizing PDP 2017 has been a team effort. We would like to thank all the members of the St. Petersburg Institute for Informatics and Automation of the Russian Academy of Sciences who have contributed so much of their time and enthusiasm to the running of PDP 2017.

We thank Anatoly Tkach, Evgeny Silla, Vasily Desnitsky, Igor Saenko, Andrey Chechulin, Elena Doynikova, Alexander Branitskiy, Andrey Fedorchenko, Mikhail Bulgakov, Maxim Kolomeec, Alexei Kushnerevich and Dmitry Levshun for hard and efficient work on organization of the PDP 2017; the St. Petersburg Institute for Informatics and Automation of the Russian Academy of Sciences for providing the setting for PDP 2017; the service agency Monomax for their support with accommodation booking and events organization. The PDP 2017 conference was supported by the grant of Russian Science Foundation (RSF) \#15-11-30029.

We wish you an enjoyable stay in St. Petersburg.

Rafael Yusupov, St. Petersburg Institute for Informatics and Automation of the Russian Academy of Sciences Igor Kotenko, St. Petersburg Institute for Informatics and Automation of the Russian Academy of Sciences PDP 2017 Organizing Committee Chairs 


\section{Conference Organization PDP 2017}

\section{Conference General Chairs}

Igor Kotenko, St. Petersburg Institute for Informatics and Automation of the Russian Academy of Sciences Yiannis Cotronis, National and Kapodistrian University of Athens

Masoud Daneshtalab, KTH Royal Institute of Technology

\section{Organizing Committee Chairs}

Rafael Yusupov, St. Petersburg Institute for Informatics and Automation of the Russian Academy of Sciences Igor Kotenko, St. Petersburg Institute for Informatics and Automation of the Russian Academy of Sciences

\section{Organizing Committee}

Anatoly Tkach

Evgeny Silla

Vasily Desnitsky

Andrey Chechulin
Igor Saenko

Elena Doynikova

Alexander Branitskiy

Andrey Fedorchenko
Mikhail Bulgakov

Maxim Kolomeec

Alexei Kushnerevich

Dmitry Levshun 


\section{Program Committees PDP 2017}

\section{Main Conference}

\section{Programme Chairs}

Igor Kotenko, St. Petersburg Institute for Informatics and Automation of the Russian Academy of Sciences Yiannis Cotronis, National and Kapodistrian University of Athens

Masoud Daneshtalab, KTH Royal Institute of Technology

\section{Programme Committee}

Marco Aldinucci

Angelos Amanatiadis

Juan L. Aragón

Mats Aspnäs

Daniele D'Agostino

Rob Baxter

Julien Bourgeois

Peter Brauer

Massimo Canonico

Zhezhe Chen

Weiwei Chen

Andrea Clematis

Angelo Corana

Donato D'Ambrosio

Marco Danelutto

Francisco De Sande

Vasily Desnitsky

Didier El Baz

Christian Engelmann

Antonella Galizia

Manoj Gaur

Daniel Garcia

Vladimir Getov

Horacio Gonzalez-Velez

Håkan Grahn

Armin Größlinger
Miaoqing Huang

Saurabh Hukerikar

Rika Ito

Adrian Jackson

Magnus Jahre

Jorn W Janneck

Gert Jervan

Gabriele Jost

Gregory Karagiorgos

Christoph Kessler

Farshad Khunjush

Peter Kilpatrick

Konrad Kloeckner

Elias Konstantinidis

Sebastien Lafond

Khalid Latif

Coromoto Leon

Francesco Leporati

Huang Letian

Diego Lopez

Konstantinos Margaritis

Luisa Massari

Massimiliano Meneghin

Nikolaos Missirlis

Koji Nakano

Smail Niar
Tomas Nordström

Salvatore Orlando

Jin Park

Raffaele Perego

Radu Prodan

Amir Rahmani

Jean Roman

Igor Saenko

Hamid Sarbazi-Azad

Georgios Ch. Sirakoulis

Amund Skavhaug

Alejandro Soba

Fengguang Song

William Spataro

Ivor Spence

Daniele Tessera

Francisco Tirado

Massimo Torquati

Giuseppe A. Trunfio

Volodymyr Turchenko

Zain Ul-Abdin

Frédéric Vivien

Roland Wismüller

Thomas Canhao $\mathrm{Xu}$

Laurence T. Yang

Vladimir Zaborovsky

\section{Special Session: GPU Computing and Many Integrated Core Computing}

\section{Programme Chair}

Didier El Baz

\section{Programme Committee}

Vincent Boyer

David Defour

Fumihiko Ino
Volodymyr Kindratenko

Bastien Plazolles

Premysl Sucha
Cornelis Vuik 
Special Session: Formal Approaches to Parallel and Distributed Systems

\section{Programme Chairs}

Simon Bliudze

Borzoo Bonakdarpour

\section{Programme Committee}

Gul Agha

Michele Amoretti

Farhad Arbab

Lacramioara Astefanoaei

Paul Attie

Roderick Bloem

Laura Bocchi

Albert Cohen

Yliès Falcone
Ludovic Henrio

Marieke Huisma

Swen Jacobs

Peter Kilpatrick

Igor Konnov

Sandeep Kulkrani

Alberto Lluch Lafuente

Frederic Loulergue

Neeraj Mittal
Anca Muscholl

Sergio Rajsbaum

Gwen Salaün

Sven Schewe

Elena Sherman

Francesco Tiezzi

Sebastien Tixeuil

Enrico Tronci

Emilio Tuosto

Special Session: Advances in High-Performance Bioinformatics, Systems and Synthetic Biology

\section{Programme Chairs}

Daniele D'Agostino

Ivan Merelli

\section{Programme Committee}

Claudia Angelini

Beretta Stefano

Yuri Pirola

Maurizio Drocco

Sandra Gesing

Fabio Tordini

Special Session: Security in Parallel, Distributed and Network-Based Computing

\section{Programme Chair}

Igor Kotenko

\section{Programme Committee}

Fabrizio Baiardi

Cataldo Basile

Julien Bourgeois

Dumitru Dan Burdescu

Andrey Chechulin

Luigi Coppolino

Miguel Correia

Herve Debar

Dennis Gamayunov

Dieter Gollmann

Stefanos Gritzalis

\author{
Alexander Grusho \\ Ming-Yuh Huang \\ Spyros Kokolakis \\ Antonio Mana \\ Fabio Martinelli \\ Gregorio Martinez Perez \\ Nikolay Moldovyan \\ Haralambos Mouratidis \\ Simin Nadjm-Tehrani \\ Nuno Neves \\ Evgenia Novikova
}

\author{
Vladimir Oleshchuk \\ Lotfi Othmane \\ Roland Rieke \\ Luigi Romano \\ Igor Saenko \\ Jose Such \\ Peter Teufl \\ Shambhu Upadhyaya \\ Brecht Wyseur \\ Ilsun You \\ Peter Zegzhda
}


Special Session: Energy Efficient Management of Parallel Systems, Platforms, and Computations

\section{Programme Chairs \\ Sebastien Lafond \\ Laurent Lefèvre \\ Jean-Marc Pierson \\ Tim Süß \\ Tuan Trinhtrinh}

\section{Programme Committee}

Attila Csaba Marosi

Georges Da Costa

Olivier Gluck

Philipp Gschwandtner
Zhiyi Huang

Nick Johnson

Jorg Keller

Lionel Morel
Lars Nagel

Maxime Pelcat

Domenico Talia

Special Session: Cloud Computing on Infrastructure as a Service and its Applications

\section{Programme Chairs}

Gabor Kecskemeti

Thierry Delaitre

Attila Kertesz

\section{Programme Committee}

Vincent Chimaobi Emeakaroha

Thomas Fahringer

Philipp Leitner

Attila Csaba Marosi

Anne-Cecile Orgerie

Radu Prodan
Ivan Rodero

Massimo Villari

Vlado Stankovski

Steven Latre

Special Session: High Performance Computing in Modeling and Simulation

\section{Programme Chairs}

William Spataro

Georgios Ch. Sirakoulis

Giuseppe A. Trunfio

Donato D'Ambrosio

\section{Programme Committee}

Angelos Amanatiadis

Gianluigi Folino

Mario Cannataro

Louis D'Alotto
Ioakeim Georgoudas

Yaroslav Sergeyev

Rocco Rongo

Pawel Topa
Marco Villani

Jaroslaw Was

Massimo Cafaro 


\section{Special Session: On-Chip Parallel and Network-Based Systems}

\section{Programme Chairs}

Nader Bagherzadeh

Masoud Daneshtalab

Masoumeh Ebrahimi

Hamid Sarbazi-Azad

\section{Programme Committee}

Hossein Asadi

Mohamed Bakhouya

Julien Bourgeois

Diana Goehringer

Somayyeh Koohi

Seung Eun Lee
Samia Loucif

Mehdi Modarressi

Siamak Mohammadi

Martin Radetzki

Fredy Rivera

Nozar Tabrizi
Hamid Zarandi

Farshad Khunjush

Piotr Dziurzanski

Farshad Safaei 


\section{Reviewers}

\section{PDP 2017}

Emmanuel Agullo

Maria Vittoria Avolio

Iman Azimi

Sakil Barbhuiya

Esha Barlaskar

Alexander Bolotov

Chongxiao Cao

Fernando Castro

Daniel Chaver

Xiuhong Chen

Sina Darabi

Patrizio Dazzi

Vincenzo De Maio

Marco L. Della Vedova

József D. Dombi

Tingxing Dong

Prokopios Drogkaris

Antoine El Hokayem

David Engdal

Antonio Flores Gil

Giordana Florimbi

Alessandro Fontanella

Juan Fumero

Stefan Ganser

Carlos Garcia Sanchez

Antonios Gasteratos

Yi Gu

Abdou Guermouche

Wajdi Hajji

Dachuan Huang

Yasuaki Ito

Majid Jalili

Tobias Kalb

Dragi Kimovski

Stefan Kronawitter

Martin Lundqvist

Tao Mao

Roland Mathà

Claudia Misale

Juan Mompeán

Andrea Morichetta

Aare Mällo

Behailu Shiferaw Negash

Zsolt Nemeth

Réda Nouacer
Pablo Padrón Santana

Karl Palmskog

Jens Rettkowski

Mohammad Sadrosadati

Nishant Saurabh

Luigi Sgaglione

Andreas Simbürger

Giuseppe Spingola

Patricia Stolf

Hongyang Sun

Daisuke Takafuji

Mojtaba Tarihi

Zisis Tsiatsikas 


\section{25th Euromicro International Conference on Parallel, Distributed and Network-Based Processing PDP 2017 Table of Contents}

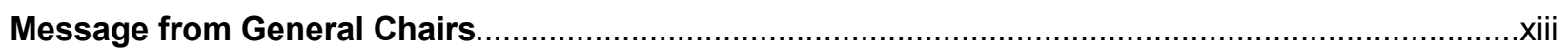

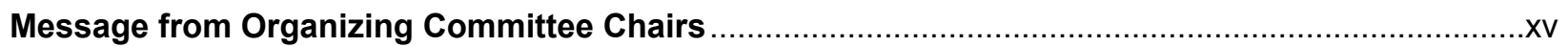

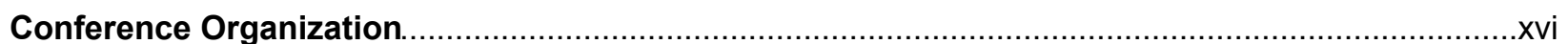

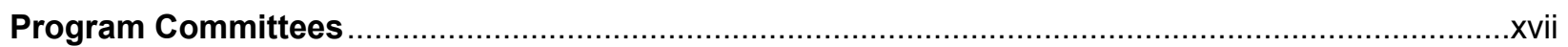

Reviewers

\section{Main Track}

A Parallel Memetic Algorithm for the Pickup and Delivery Problem with Time

Windows .1 Jakub Nalepa and Miroslaw Blocho

Cloud Storage Cost Modeling for Cryptographic File Systems Mauro Storch and César A. F. de Rose

An FPGA-based In-NIC Cache Approach for Lazy Learning Outlier Filtering Ami Hayashi and Hiroki Matsutani

A Parallel Variant of LDSieve for the SVP on Lattices

Artur Mariano, Thijs Laarhoven, and Christian Bischof

High Performance I/O for Seismic Wave Propagation Simulations

Francieli Zanon Boito, Jean Luca Bez, Fabrice Dupros, Mario A. R. Dantas,

Philippe O. A. Navaux, and Hideo Aochi

Modelling Low Power Compute Clusters for Cloud Simulation

Gabor Kecskemeti, Wajdi Hajji, and Fung Po Tso

MERCURY: A Transparent Guided I/O Framework for High Performance I/O

Stacks

Giuseppe Congiu, Matthias Grawinkel, Federico Padua, James Morse,

Tim Süß, and André Brinkmann 
Parallel Satisfiability Solver Based on Hybrid Partitioning Method

Tarek Menouer and Souheib Baarir

Elastic Scaling for Distributed Latency-Sensitive Data Stream Operators

Tiziano de Matteis and Gabriele Mencagli

Parallelization of Machine Learning Applied to Call Graphs of Binaries

for Malware Detection

Robert Searles, Lifan Xu, William Killian, Tristan Vanderbruggen,

Teague Forren, John Howe, Zachary Pearson, Corey Shannon,

Joshua Simmons, and John Cavazos

Fault-Tolerant Parallel Execution of Workflows with Deadlines

Patrick Eitschberger and Jörg Keller

A Rapid Data Communication Exploration Tool for Hybrid CPU-FPGA

Architectures

Mariem Makni, Smail Niar, Mouna Baklouti, Guanwen Zhong, Tulika Mitra, and Mohamed Abid

Efficient Regional Congestion Awareness (ERCA) for Load Balance

with Aggregated Congestion Information

Sheng Xu, Jie Wu, Binzhang Fu, Mingyu Chen, and Lixin Zhang

Decentralized Management of Random Walks over a Mobile Phone Network Árpád Berta and Márk Jelasity

Softening Up the Network for Scientific Applications 108

Celio Trois, Luis C. E. de Bona, Marcos D. Del Fabro, Magnos Martinello,

Sarvesh Bidkar, Reza Nejabati, and Dimitra Simeonidou

TWINS: Server Access Coordination in the I/O Forwarding Layer

Jean Luca Bez, Francieli Zanon Boito, Lucas M. Schnorr,

Philippe O. A. Navaux, and Jean-François Méhaut

A Region-Based Approach to Pipeline Parallelism in Java Programs

on Multicores

Yang Wang and Kenneth B. Kent

CloudMapper: A Model-Based Framework for Portability of Cloud Applications

Consuming PaaS Services

Riccardo Munisso and Adriana E. Chis

Dynamic Load Balancing of Monte Carlo Particle Transport Applications

on HPC Clusters

Thomas Gonçalves, Frédéric Desprez, and Jean-François Méhaut

NoSQL Database Record Versions Processing Model

Aleksey Burdakov, Uriy Grigorev, Eugene Ttsviashchenko, and Andrey Ploutenko 
Efficient Multi-core AUTOSAR-Platform Based on an Input/Output Gateway

Core

Moisés Urbina and Roman Obermaisser

Transforming Procedural Code for Streaming Environments

Michal Brabec and David Bednárek

Hierarchal Placement of Smart Mobile Access Points in Wireless Sensor

Networks Using Fog Computing

Amin Majd, Golnaz Sahebi, Masoud Daneshtalab, Juha Plosila, and Hannu Tenhunen

Mobile Application Testing on Clouds: Challenges, Opportunities

and Architectural Elements

Miguel G. Xavier, Kassiano J. Matteussi, Gabriel R. França, Wagner P. Pereira,

and Cesar A. F. de Rose

On the Overhead of Topology Discovery for Locality-Aware Scheduling in HPC

Brice Goglin

Resource Management for Mobile Publish/Subscribe Systems

Fatma Abdennadher and Maher Ben Jemaa

Flexible Representation of loT Sensors for Cloud Simulators

Andras Markus, Gabor Kecskemeti, and Attila Kertesz

Parallelizing Soft-Synths with Soft Real-Time Requirements

Ede Cameron and Dhrubajyoti Goswami

Characterizing Performance and Cache Impacts of Code Multi-versioning

on Multicore Architectures

Peter Zangerl, Peter Thoman, and Thomas Fahringer

Using Bootstraping Principles of Contemporary P2P File-Sharing Protocols

in Large-Scale Grid Computing Systems

Josef Gattermayer and Pavel Tvrdík

Evaluating Concurrency Throttling and Thread Packing on SMT Multicores

Marco Danelutto, Tiziano de Matteis, Daniele de Sensi, and Massimo Torquati

Performance of Krylov Subspace Methods for Symmetric Matrices in Hybrid

Parallelization

Kuniyoshi Abe and Seiji Fujino

Data Race Detection by Understanding Synchronization Relationships

of Thread Segments

Zhiyuan Shao, Jian Peng, and Hai Jin

Warstack: Improving LLC Replacement for NVM with a Writeback-Aware

Reuse Stack

Hanfeng Qin and Hai Jin 
Energy-Efficient and Portable Least Squares Prediction for Image Coding on a Mobile GPU

Pedro Cordeiro, Gabriel Falcao, Patricio Domingues, Nuno Rodrigues, and Sergio Faria

Educational Multiprocessor Simulator "E14" and Its Usage for Expanding the Formula of Amdahl's Law

Evgeny A. Eremin

TORMENT OpenACC2016: A Benchmarking Tool for OpenACC Compilers Daniel Barba, Arturo Gonzalez-Escribano, and Diego R. Llanos

A QoS Bandwidth Allocation Method for Coexistence of Wireless Body Area Networks 251

Da-Ren Chen

\section{GPU Computing and Many Integrated Core Computing}

Asynchronous Power Flow on Graphic Processing Units

Manuel Marin, David Defour, and Federico Milano

GPU-based Bio-inspired Model for Solving Association Rules Mining Problem

Youcef Djenouri, Ahcene Bendjoudi, Djamel Djenouri, and Marco Comuzzi

On the Evaluation of Energy-Efficient Deep Learning Using Stacked

Autoencoders on Mobile GPUs

G. Falcao, L. A. Alexandre, J. Marques, X. Frazao, and J. Maria

Efficient Parallelization of Motion Estimation for Super-Resolution

Elisa Marenzi, Andrea Carrus, Giovanni Danese, Francesco Leporati, and Gustavo Marrero Callicò

\section{Formal Approaches to Parallel and Distributed Systems}

Model Checking Geographically Distributed Interlocking Systems Using UMC

Alessandro Fantechi, Anne E. Haxthausen, and Michel Bøje Randahl Nielsen

Automatic Refinement for Event-B through Annotated Patterns 287

Badr Siala, Jean-Paul Bodeveix, Mamoun Filali, and Mohamed Tahar Bhiri

Analysing Message Numbers in Actor Systems

Marco Grebe, Tilman Lacko, and Rita Loogen 


\section{Advances in High-Performance Bioinformatics, Systems and Synthetic Biology}

CUDA-Sankoff: Using GPU to Accelerate the Pairwise Structural RNA

Alignment

Daniel Sundfeld, Jakob H. Havgaard, Jan Gorodkin, and Alba C. M. A. de Melo

Implementing a Space-Aware Stochastic Simulator on Low-Power

Architectures: A Systems Biology Case Study

Lucia Morganti, Elena Corni, Andrea Ferraro, Daniele Cesini,

Daniele D'Agostino, and Ivan Merelli

Low-Power Architectures for miRNA-Target Genome Wide Analysis

Stefano Beretta, Lucia Morganti, Elena Corni, Andrea Ferraro, Daniele Cesini,

Daniele D'Agostino, Luciano Milanesi, and Ivan Merelli

\section{Security in Parallel, Distributed and Network-Based Computing}

Sound Covert: A Fast and Silent Communication Channel through the Audio

Buffer

Ofir Shwartz and Yitzhak Birk

A Distributed Framework for Collaborative and Dynamic Analysis of Android

Malware

Mario Faiella, Antonio La Marra, Fabio Martinelli, Francesco Mercaldo,

Andrea Saracino, and Mina Sheikhalishahi

Parallel Processing of Big Heterogeneous Data for Security Monitoring of loT

Networks

Igor Saenko, Igor Kotenko, and Alexey Kushnerevich

Privacy-Preserving Location-Proximity for Mobile Apps

Simonas Stirbys, Omar Abu Nabah, Per Hallgren, and Andrei Sabelfeld

CVSS-based Probabilistic Risk Assessment for Cyber Situational Awareness

and Countermeasure Selection

Elena Doynikova and Igor Kotenko

Analysing the Impact of a DDoS Attack Announcement on Victim Stock Prices

Abhishta, Reinoud Joosten, and L. J. M. Nieuwenhuis

Cloudifying Critical Applications: A Use Case from the Power Grid Domain

F. Campanile, L. Coppolino, S. D’Antonio, L. Lev, G. Mazzeo, L. Romano,

L. Sgaglione, and F. Tessitore

Using S-Rules to Fire Dynamic Countermeasures

F. Baiardi, J. Lipilini, and F. Tonelli 
A Formal Model of Patrolling Game and its Agent-Based Simulation Using Jason

Amelia Bădică, Costin Bădică, Cătălina Sitnikov, and Florin Leon

Behavior Analysis for Safety and Security in Automotive Systems

Roland Rieke, Marc Seidemann, Elise Kengni Talla, Daniel Zelle,

and Bernhard Seeger

A Survey on Fake Entities as a Method to Detect and Monitor Malicious

Activity 386

Sampsa Rauti and Ville Leppänen

Towards Stronger Data Security in an eID Management Infrastructure

Diana Berbecaru, Andrea Atzeni, Marco de Benedictis, and Paolo Smiraglia

Surveying and Analyzing Access Control Models in Cloud Computing

Mariem Bouchaala, Cherif Ghazel, Leila Azouz Saidane, and Farouk Kamoun

\section{Energy Efficient Management of Parallel Systems, Platforms and Computations}

Core Level Utilization for Achieving Energy Efficiency in Heterogeneous

Systems

Hergys Rexha, Simon Holmbacka, and Sébastien Lafond

Balancing the Use of Batteries and Opportunistic Scheduling Policies

for Maximizing Renewable Energy Consumption in a Cloud Data Center

Yunbo Li, Anne-Cécile Orgerie, and Jean-Marc Menaud

How Much Energy Can Green HPC Cloud Users Save?

David Guyon, Anne-Cécile Orgerie, Chrtistine Morin, and Deb Agarwal

Asymmetric Crown Scheduling

Manfred Torggler, Jörg Keller, and Christoph Kessler

epEBench: True Energy Benchmark

Simon Holmbacka and Robert Müller

\section{Cloud Computing on Infrastructure as a Service and Its Applications}

Virtual Machine Boot Time Model

Thuy Linh Nguyen and Adrien Lebre

Efficient Bottleneck Detection in Stream Process System Using Fuzzy Logic

Model

Yanlong Zhai and Wu Xu

Analysing the Performance Instability Correlation with Various Workflow

and Cloud Parameters

Sasko Ristov, Roland Mathá, and Radu Prodan 
Network-Aware VM Migration Heuristics for Improving the SLA Violation of Multi-Tier Web Applications in the Cloud

Amir Hossein Borhani, Terence Hung, Bu-Sung Lee, Zheng Qin, and Zahra Bagheri

Transparent Execution of Task-Based Parallel Applications in Docker with COMP Superscalar

Victor Anton, Cristián Ramón-Cortes, Jorge Ejarque, and Rosa M. Badia

An Intra-Cloud Networking Performance Evaluation on CloudStack

Environment

Adriano Vogel, Dalvan Griebler, Claudio Schepke, and Luiz Gustavo Fernandes

HPC Application Performance and Cost Efficiency in the Cloud

Eduardo Roloff, Matthias Diener, Luciano Paschoal Gaspary,

and Philippe O. A. Navaux

Use Cases towards a Decentralized Repository for Transparent and Efficient

Virtual Machine Operations

Radu Prodan, Thomas Fahringer, Dragi Kimovski, Gabor Kecskemeti,

Attila Csaba Marosi, Vlado Stankovski, Jonathan Becedas, Jose Julio Ramos,

Craig Sheridan, Darren Whigham, and Carlos Rodrigo Rubia Marcos

Coherent Application Delivery on Hybrid Distributed Computing Infrastructures

of Virtual Machines and Docker Containers

Germán Moltó, Miguel Caballer, Alfonso Pérez, Carlos de Alfonso,

and Ignacio Blanquer

\section{High Performance Computing in Modeling and Simulation}

A Tracking Algorithm for Particle-Like Moving Objects

Davide Spataro, Paola Arcuri, Alessio de Rango, William Spataro,

Donato D'Ambrosio, and Alice Mari

A GPU Implemented 3F Cellular Automata-Based Model for a 2D Evacuation

Simulation Pattern

Isaac Koumis, loakeim G. Georgoudas, Giuseppe A. Trunfio, Jarosław Wąs,

and Georgios Ch. Sirakoulis

Parallel Execution of Cellular Automata through Space Partitioning: The

Landslide Simulation Sciddicas3-Hex Case Study

Andrea Giordano, Alessio de Rango, Davide Spataro, Donato D'Ambrosio,

Carlo Mastroianni, Gianluigi Folino, and William Spataro

From Python Scripting to Parallel Spatial Modeling: Cellular Automata

Simulations of Land Use, Hydrology and Pest Dynamics

Jesús Carabaño and Jan Westerholm 
Parallel and Cloud-Based Analysis of Omics Data: Modelling and Simulation in Medicine

Giuseppe Agapito, Barbara Calabrese, Pietro H. Guzzi, Gionata Fragomeni,

Giuseppe Tradigo, Pierangelo Veltri, and Mario Cannataro

High Performant Simulations of Cerebellar Golgi Cells Activity

Giordana Florimbi, Emanuele Torti, Giovanni Danese, and Francesco Leporati

A Comparative Analysis of Data-Driven Consolidation Policies

for Energy-Efficient Clouds

Albino Altomare and Eugenio Cesario

A Peer to Peer Approach to Efficient High Performance Computing

Nunziato Cassavia, Sergio Flesca, Michele lanni, Elio Masciari,

Giuseppe Papuzzo, and Chiara Pulice

Noise Inspector Tool

Gladys Utrera, Jordi Fornes, and Jesus Labarta

Task Packing: Getting the Best from MPI Unbalanced Applications

Gladys Utrera, Montse Farreras, and Jordi Fornes

\section{On-Chip Parallel and Network-Based Systems}

Global Adaptation for Energy Efficiency in Multicore Architectures

Alina Lenz, Tobias Pieper, and Roman Obermaisser

Register-Exchange Based Connection Allocator for Circuit Switching NoCs

Yong Chen, Emil Matus, and Gerhard P. Fettweis

Buffer-Aware Analysis for Worst-Case Traversal Time of Real-Time Traffic

over RRA-based NoCs 567

Meng Liu, Matthias Becker, Moris Behnam, and Thomas Nolte

Cache Energy Management through Dynamic Reconfiguration Approach

in Opto-Electrical NoC

Saba Jamilan, Meisam Abdollahi, and Siamak Mohammadi

Compiler-Enhanced Reliability for Network-on-Chip Architectures

Muhammad Aditya Sasongko, Haluk Rahmi Topcuoglu, Sanem Arslan,

and Mahmut Taylan Kandemir

Multi-objective Task Mapping Approach for Wireless NoC in Dark Silicon Age

Amin Rezaei, Dan Zhao, Masoud Daneshtalab, and Hai Zhou

3D-AMAP: A Latency-Aware Task Mapping onto 3D Mesh-Based NoCs

with Partially-Filled TSVs

Hesamedin Ziaeeziabari and Ahmad Patooghy

Author Index .598 